\title{
Comparison of specific gravity analysis of feline and canine urine, using five refractometers, to pycnometric analysis and total solids by drying
}

\author{
HW Tvedten ${ }^{*}$, H Ouchterlony ${ }^{\dagger}$ and IE Lilliehöök ${ }^{\ddagger}$
}

${ }^{*}$ Clinical Sciences, Faculty of Veterinary Medicine and Animal Sciences, Swedish University of Agricultural Sciences.

tInstitution for Women and Children's Health, Uppsala University.

期iversity Animal Hospital, Swedish University of Agricultural Sciences, Uppsala, Sweden.

\Author for correspondence: Email: tvedten@msu.edu

Supplementary Table 1. Specific gravity results from analysis of pure solutions of creatinine, $\mathrm{NaCl}$ and urea using five different refractometers ${ }^{a}$. Note that the chemical form of creatinine is a light powder that is difficult to weigh and dispense accurately, therefore some error in concentrations of creatinine are possible.

\begin{tabular}{|l|c|c|c|c|c|c|c|}
\hline Substance & Concentration & Atago 1 & Rei 2 & Rei 3 & Vet Dog & Vet Cat & Atago Cat \\
\hline Creatinine & $100 \mathrm{~g} / \mathrm{L}$ & 1.048 & $>1.035$ & $>1.035$ & 1.045 & 1.042 & 1.041 \\
\hline Creatinine & $75 \mathrm{~g} / \mathrm{L}$ & 1.040 & 1.035 & 1.035 & 1.034 & 1.030 & 1.030 \\
\hline Creatinine & $66.7 \mathrm{~g} / \mathrm{L}$ & 1.037 & 1.032 & 1.032 & 1.031 & 1.026 & 1.026 \\
\hline Creatinine & $50 \mathrm{~g} / \mathrm{L}$ & 1.030 & 1.024 & 1.024 & 1.023 & 1.019 & 1.019 \\
\hline Creatinine & $33 \mathrm{~g} / \mathrm{L}$ & 1.024 & 1.017 & 1.017 & 1.017 & 1.014 & 1.014 \\
\hline Creatinine & $25 \mathrm{~g} / \mathrm{L}$ & 1.020 & 1.014 & 1.013 & 1.013 & 1.010 & 1.010 \\
\hline Creatinine & $10 \mathrm{~g} / \mathrm{L}$ & 1.010 & 1.005 & 1.005 & 1.005 & 1.004 & 1.004 \\
\hline $\mathrm{NaCl}$ & $100 \mathrm{~g} / \mathrm{L}$ & 1.045 & $>1.035$ & $>1.035$ & 1.040 & 1.036 & 1.036 \\
\hline $\mathrm{NaCl}$ & $75 \mathrm{~g} / \mathrm{L}$ & 1.037 & 1.033 & 1.033 & 1.031 & 1.027 & 1.027 \\
\hline $\mathrm{NaCl}$ & $66.7 \mathrm{~g} / \mathrm{L}$ & 1.035 & 1.029 & 1.029 & 1.028 & 1.024 & 1.024 \\
\hline $\mathrm{NaCl}$ & $50 \mathrm{~g} / \mathrm{L}$ & 1.029 & 1.023 & 1.022 & 1.022 & 1.018 & 1.018 \\
\hline $\mathrm{NaCl}$ & $33 \mathrm{~g} / \mathrm{L}$ & 1.020 & 1.015 & 1.015 & 1.015 & 1.012 & 1.012 \\
\hline $\mathrm{NaCl}$ & $25 \mathrm{~g} / \mathrm{L}$ & 1.016 & 1.012 & 1.012 & 1.011 & 1.009 & 1.009 \\
\hline $\mathrm{NaCl}$ & $10 \mathrm{~g} / \mathrm{L}$ & 1.010 & 1.005 & 1.005 & 1.004 & 1.004 & 1.004 \\
\hline Urea & $200 \mathrm{~g} / \mathrm{L}$ & $>1.050$ & $>1.035$ & $>1.035$ & 1.060 & 1.057 & 1.057 \\
\hline Urea & $150 \mathrm{~g} / \mathrm{L}$ & 1.048 & $>1.035$ & $>1.035$ & 1.046 & 1.042 & 1.042 \\
\hline Urea & $100 \mathrm{~g} / \mathrm{L}$ & 1.038 & 1.034 & 1.034 & 1.032 & 1.028 & 1.028 \\
\hline Urea & $50 \mathrm{~g} / \mathrm{L}$ & 1.025 & 1.018 & 1.018 & 1.017 & 1.014 & 1.014 \\
\hline Urea & $25 \mathrm{~g} / \mathrm{L}$ & 1.015 & 1.009 & 1.009 & 1.009 & 1.007 & 1.007 \\
\hline Urea & $10 \mathrm{~g} / \mathrm{L}$ & 1.009 & 1.004 & 1.004 & 1.003 & 1.003 & 1.003 \\
\hline Urea & $2 \mathrm{~g} / \mathrm{L}$ & 1.003 & 1.001 & 1.001 & 1.000 & 1.000 & 1.001 \\
\hline
\end{tabular}

${ }^{a}$ Atago 1 was a Uricon refractometer (Atago Corp, Tokyo Japan); Rei 2 was a Reichert TS meter, Rei 3 was a Leica TS meter (both were Model 10400A Reichert TS meters; Cambridge Instruments Inc, Buffalo NY, USA); Vet Cat and Vet Dog were the two scales, for cats and dogs, respectively, for a Model 10436 AO Veterinary refractometer (AO Scientific Instruments, Buffalo NY, USA); Atago Cat was a digital Atago 'Pocket' PAL-USG Cat refractometer (Atago Corp). 
